\title{
A nonlinear neural population coding theory of quantum cognition and decision making
}

\author{
Taiki Takahashi ${ }^{1}$, Taksu Cheon ${ }^{2}$ \\ ${ }^{1}$ Department of Behavioral Science, Center for Experimental Research in Social Sciences, Hokkaido University, Sapporo, Japan \\ ${ }^{2}$ Laboratory of Physics, Kochi University of Technology, Kochi, Japan \\ Email: taikitakahashi@gmail.com
}

Received 15 August 2012; revised 27 September 2012; accepted 12 October 2012

\begin{abstract}
Mathematical frameworks of quantum theory have recently been adopted in cognitive and behavioral sciences, to explain the violations of normative decision theory and anomalies in cognition. However, to date, no study has attempted to explore neural implementations of such "quantum-like" information processing in the brain. This study demonstrates that neural population coding of information with nonlinear neural response functions can account for such "quantum" information processing in decision-making and cognition. It is also shown that quantum decision theory is a special case of more general population vector cording theory. Future applications of the present theory in the rapidly evolving field of "psychophysical neuroeconomics” are also discussed.
\end{abstract}

Keywords: Quantum Probability; Psychophysics; Neuroeconomics; Population Coding

\section{INTRODUCTION}

Recent years witnessed a rapid growing of the applications of mathematical frameworks of quantum physics [1-3] and quantum information theory [4] in psychology, cognitive science, behavioral science, and economics [5-11]. One of the advantages of the utilization of "quantum" theoretical frameworks is that quantum probability theory can describe the violation of some classical probability laws such as the law of total probability [10]. Although we claimed the importance of the explorations of possible cognitive mechanisms underlying such "quantum"- like behavior in human decision and cognition $[7,8]$, no study to date addressed this issue. This point is important, because it is not very probable that quantum mechanical effects appear in the brain under normal physical conditions at body temperature [12].

We now show a simple example of the violation of classical probability laws in human decision making and cognition (corresponding to the violation of the Sure- thing Principle in decision theory proposed by [13]). The law of total probability states that

$$
P\left(a_{1}\right)=P\left(b_{0}\right) P\left(a_{1} \mid b_{0}\right)+P\left(b_{1}\right) P\left(a_{1} \mid b_{1}\right)
$$

where $P\left(x_{i}\right)(x=a, b ; i=0,1)$ is a probability at which event $x_{i}$ occurs and $P\left(x_{i} \mid y_{j}\right)$ is a conditional probability of event $x_{i}$ given event $y_{j} \quad(y=a, b ; j=0,1)$. Mathematical psychologists Tversky and colleagues experimentally demonstrated that humans violate the law of total probability in their probabilistic choice (referred to as "disjunction effect", $[14,15])$ and probability judgment (referred to as "conjunction fallacy", [16]). The simplest quantum formalism for modeling the violation of the law of total probability is [9]:

$$
\begin{aligned}
& P\left(a_{1}\right)=P\left(b_{0}\right) P\left(a_{1} \mid b_{0}\right)+P\left(b_{1}\right) P\left(a_{1} \mid b_{1}\right) \\
& +2 \sqrt{P\left(a_{1}\right) P\left(b_{0}\right) P\left(a_{1} \mid b_{0}\right) P\left(a_{1} \mid b_{1}\right)} \cos \theta
\end{aligned}
$$

Where $\theta$ is a "quantum phase" parameterizing the degree of the violation ("quantum interference") of the law of total probability (see [7,8], for more complete quantum formalism with "composite" system setting). It is to be noted that Eq.1-2 is obtained through "Born's rule" in quantum probability theory (see [1], for a standard reference of quantum theory).

\section{POPULATION CODING THEORY OF QUANTUM COGNITION AND DECISION-MAKING}

In neuroscience of sensory and motor systems, it has been established that information of sensory input and motor output is, in many cases, encoded at the neuronal population level rather than individual single neuron level [17-20]. Let us start our current investigation into the neural foundation of quantum decision theory from this empirical observation. Suppose that (scalar) physical input (or output) signal (from sensory organs or to motor systems) activates a neuronal ensemble (a population vector) consisting of $n$ neurons, even when the intensity (magnitude) of input/output signal is a single real scalar 
parameter. The physical input to (or output from) the ensemble of $n$ neurons ("population state vector") can be expressed in a vector form as:

$$
p x=\left(p x_{1}, p x_{2}, \cdots, p x_{n}\right)
$$

where $p x_{1}$ is a real number which indicates the input to single neuron $i$ in the neural population (ensemble) consisting of the $n$ neurons.

The important point here is that even when the signal is a (real) scalar number (e.g. time, probability, the intensity of sound and light), the corresponding input to the neural ensemble system is in a vector form (of which components are real numbers) in Eq.2-1, in mathematical terms.

Then, the $n$ neurons' response vector for the neural population is

$$
x=\left(f\left(p x_{1}\right), f\left(p x_{2}\right), \ldots, f\left(p x_{n}\right)\right)=\left(x_{1}, \ldots, x_{n}\right),
$$

where each component $f\left(p x_{i}\right)=x_{i}(i$ is a positive integer no larger than $n$ ) of the vector $x$ is (real scalar) each single neuron's response (e.g., a change in firing rate $[\mathrm{Hz}]$, the concentration of the product of induced biochemical reactions $[\mathrm{nmol} / \mathrm{L}]$, note that $x_{i}$ could be negative) of neuron $i$ in the neural population consisting of $n$ neurons. Notably, the function $f$ is generally nonlinear (see standard neuroscience textbooks such as [21]). We can now assume that the real scalar "intensity" $\Phi$ of the neuronal ensemble's response to $p x$ (a total sum of all the $n$ neurons' activations) is:

$$
\Phi(p x)=\sum_{i=1}^{n} x_{i}=\sum_{i=1}^{n} f\left(p x_{i}\right)
$$

This assumption is natural in that the result of the neural population activity may be, for instance, an increase in the level of a specific type of some neurotransmitters (e.g., glutamate, GABA, dopamine and serotonin) or hormones (cortisol, testosterone, and oxytocin) in the brain region containing the neural ensemble consisting of $n$ neurons.

Note that, as accumulating neurophysiological and psychophysical evidence suggests, this intensity of neural populational response may linearly correspond to subjective (or psychological) intensity $\Psi$ of the physical (scalar) stimulus input [19]:

$$
\Psi(p x)=k \Phi(p x)
$$

Let us then consider two distinct types of the population state vectors (input or output vectors to the neural ensemble, note that this "state" is a state of input/output, not the state of neuronal responses) $p x$ and $p y$ and a linear combination of the two population state vectors (with real weighting coefficients $c_{1}$ and $c_{2}$ ):

$$
p z:=c_{1} p x+c_{2} p y
$$

Here we can ask, what is the physical intensity of the neuronal ensemble's response to the linearly-combined input (or output) state vector $p z$ ? The answer is:

$$
\begin{aligned}
\Phi(p z) & =\Phi\left(c_{1} p x+c_{2} p z\right) \\
& =\sum_{i=1}^{n}\left(c_{1} p x_{i}+c_{2} p y_{i}\right)
\end{aligned}
$$

Here we should notice that

$$
\Phi\left(c_{1} p x+c_{2} p y\right) \neq \mathrm{c}_{1} \Phi(p x)+\mathrm{c}_{2} \Phi(p y)
$$

The reason for this inequality is that $f$ is generally a non-linear function. As we will see later, the violation of the linear additivity of neural ensemble's response shown by Eq.2-7 is the mathematical root of the "quantum interference" in human decision-making and cognition.

For more intuitive understanding, let us assume that $f$ is a power function corresponding to a psychophysical quantity (such as subjective probability and preference): $f(x)=x^{s}$. In other words, we here assume Stevens' power law in psychophysics [22]. Then, Eq.2-3 reduces to

$$
\Phi\left(p x_{1}, p x_{2}, \cdots, p x_{n}\right)=\sum_{i=1}^{n}\left(p x_{i}\right)^{s}
$$

Then, the subjective quantity from the input (or output) state vector is (from Eq.2-4)

$$
\begin{aligned}
\Psi(p x) & =k \Phi\left(p x_{1}, p x_{2}, \cdots, p x_{n}\right) \\
& =k \sum_{i=1}^{n}\left(p x_{i}\right)^{s}
\end{aligned}
$$

When we consider the special case of $s=2$ with setting $k=1$ (without losing generality, because psychophysical quantity $\Psi$ is in an arbitrary unit), Eq.2-9 reduces to

$$
\Psi(p x)=\sum_{i=1}^{n}\left(p x_{i}\right)^{2}=\|p x\|
$$

Here we adopted the standard definition of the "norm" (length) of the vector: $\|p x\|$ in linear algebra. In this case when $s=2$, let us again consider a linear combination ("superposed") neural populational input (or output) state $p z=c_{1} p x+c_{2} p y$ (defined in Eq.2-5). The subjective quantity (with Stevens' exponent $s=2$ ) induced by this superposed state is:

$$
\begin{aligned}
& \Psi\left(p z=c_{1} p x+c_{2} p y\right)=\left(c_{1}\right)^{2}\|p x\|^{2} \\
& +\left(c_{2}\right)^{2}\|p y\|^{2}+2(p x, p y)
\end{aligned}
$$

(the last term is an inner product of $p x$ and $p y$ ).

$$
\begin{aligned}
& =\left(c_{1}\right)^{2}\|p x\|^{2}+\left(c_{2}\right)^{2}\|p y\|^{2} \\
& +2\left(c_{1}\right)\left(c_{2}\right)\|p x\|\|p y\| \cos \theta
\end{aligned}
$$

where $\theta$ is an angle between vectors $p x$ and $p y$.

When we put $\Psi(p z)=P\left(a_{1}\right), \quad\left(c_{1}\right)^{2}=P\left(b_{0}\right)$, $\left(c_{2}\right)^{2}=P\left(b_{1}\right),\|p x\|^{2}=P\left(a_{1} \mid b_{0}\right)$, and $\|p y\|^{2}=P\left(a_{1} \mid b_{1}\right)$, Eq.2-11 is the same as Eq.1-2 which 
often appears in quantum decision theory $[9,10]$. Taken together, it can be concluded that so-called "quantum decision theory" is a special case of more general nonlinear population coding theory of neural information (i.e., Eq.2-1 and 2-3) in which Stevens' exponent is fixed at $s$ $=2$. When $s$ is an integer larger than 2, there appears more interference terms. It should further be noted that the present theory removes the necessity of quantum physical effect (and associated complex-numbered vectors in the Hilbert space) in the human brain in explaining the seemingly "quantum-like" phenomena in human cognition and decision making. Also, psychophysical experiment demonstrated that subjective intensity of muscle force follows Stevens' power law with the exponent $s=1.7$

(http://www.cis.rit.edu/people/faculty/montag/vandplite/ pages/chap 6/ch6p10.html) which is close to 2, supporting our present hypothesis on human choice behavior.

\section{IMPLICATIONS OF THE PRESENT THEORY TO NEUROECONOMICS AND DECISION NEUROSCIENCE}

Rapid advances in neuroeconomics suggest the importance of psychophysical considerations for proper theories in decision neuroscience (we can call it "psychophysical neuroeconomics", [23-26]). For instance, anomalies in human decision making (i.e., deviations from normative decision theory or axioms in microeconomics) such as preference reversal over time in intertemporal choice has been explained by nonlinearity of subjective time in terms of physical time [25-28]. Therefore, future studies in neuroeconomics and decision neuroscience should incorporate the nonlinearity arising from population vector cording of decision parameters (e.g., utility function, psychological time, subjective probability, probability weighting function), by combining neuroeconomic theory and quantum theory of cognition and decision.

\section{ACKNOWLEDGEMENTS}

The research reported in this paper was supported by a grant from the Grant-in-Aid for Scientific Research (Innovative Areas, 23118001; Adolescent Mind \& Self-Regulation) from the Ministry of Education, Culture, Sports, Science and Technology of Japan.

\section{REFERENCES}

[1] Dirac, P.A.M. (1982) The principles of quantum mechanics. Oxford University Press, Oxford.

[2] Sakurai, J.J. (1994) Modern quantum mechanics. Pearson Education Inc., New Jersey.

[3] Peres, A. (1995) Quantum theory: Concepts and methods. Fundamental Theories of Physics, Dordrecht.
[4] Nielsen, M.A. and Chuang, I.L. (2000) Quantum computation and quantum information. Cambridge University Press, Cambridge.

[5] Busemeyer, J.R., Pothos, E.M., Franco, R. and Trueblood, J. (2011) A quantum theoretical explanation for probability judgment errors. Psychological Review, 118, 193-218. doi: $10.1037 / \mathrm{a} 0022542$

[6] Busemeyer, J.R. and Bruza, P.D. (2012) Quantum models of cognition and decision. Cambridge University Press, Cambridge. doi:10.1017/CBO9780511997716

[7] Cheon, T. and Takahashi, T. (2010) Interference and inequality in quantum decision theory. Physics Letters A, 375, 100-104. doi:10.1016/i.physleta.2010.10.063

[8] Cheon, T. and Takahashi, T. (2012) Quantum phenomenology of conjunction fallacy. Journal of Physical Society of Japan, 81, 104-801. doi:10.1143/JPSJ.81.104801

[9] Franco, R. (2009) The conjunctive fallacy and interference effects. Journal of Mathematical Psychology, 53, 415422. doi:10.1016/j.jmp.2009.02.002

[10] Khrennikov, A. (2010) Ubiquitous quantum structure. Springer, Berlin. doi:10.1007/978-3-642-05101-2

[11] Yukalov, V. and Sornette, D. (2010) Decision theory with prospect interference and entanglement. Theory and Decision, 68, 1-46.

[12] Koch, C. and Hepp, K. (2006) Quantum mechanics in the brain. Nature, 440, 611. doi:10.1038/440611a

[13] Savage, L.J. (1954) The foundations of statistics. Wiley Publications, New York.

[14] Tversky, A. and Shafir, E. (1992) The disjunction effect in choice under uncertainty. Psychological Science, 3, 305-309. doi:10.1111/j.1467-9280.1992.tb00678.x

[15] Shafir, E. and Tversky, A. (1992) Thinking through uncertainty: Nonconsequential reasoning and choice. Cognitive Psychology, 24, 449-474. doi:10.1016/0010-0285(92)90015-T

[16] Tversky, A. and Kahneman, D. (1983) Extension versus intuitive reasoning: The conjunctionfallacy in probability judgment. Psychological Review, 90, 293-315. doi:10.1037/0033-295X.90.4.293

[17] Averbeck, B.B. and Lee, D. (2004) Coding and transmission of information by neural ensembles. Trends in Neuroscience, 27, 225-230. doi:10.1016/j.tins.2004.02.006

[18] Bensmaia, S.J. (2008) Tactile intensity and population codes. Behavioural Brain Research, 190, 165-173. doi:10.1016/j.bbr.2008.02.044

[19] Johnson, K.O., Hsiao, S.S. and Yoshioka, T. (2002) Neural coding and the basic law of psychophysics. Neuroscientist, 8, 111-121. doi:10.1177/107385840200800207

[20] Georgopoulos, A.P., Schwartz, A.B. and Kettner, R.E. (1986) Neuronal population coding of movement direction. Science, 233, 1416-1419. doi:10.1126/science.3749885

[21] Kandel, E., Schwartz, J. and Jessell, T. (2000) Principles of neural science. McGraw-Hill, New York.

[22] Stevens, S.S. (1957) On the psychophysical law. Psychological Review, 64, 153-181. doi:10.1037/h0046162 
[23] Takahashi, T. (2009) Theoretical frameworks for neuroeconomics of intertemporal choice. Journal of Neuroscience, Psychology, and Economics, 2, 75-90. doi: $10.1037 / \mathrm{a} 0015463$

[24] Takahashi, T. (2011) Psychophysics of the probability weighting function. Physica A, 390, 902-905. doi:10.1016/j.physa.2010.10.004

[25] Takahashi, T., Oono, H. and Radford, M.H.B. (2008) Psychophysics of time-perception and intertemporal choice models. Physica A, 387, 2066-2074. doi:10.1016/j.physa.2007.11.047

[26] Han, R. and Takahashi, T. (2012) Psychophysics of valuation and time perception in temporal discounting of gain and loss. Physica A, 391, 6568-6576. doi:10.1016/j.physa.2012.07.012

[27] Takahashi, T. (2005) Loss of self-control in intertemporal choice may be attributable to logarithmic time-perception. Medical Hypotheses, 65, 691-693. doi:10.1016/j.mehy.2005.04.040

[28] Zauberman, G., Kim, B.K., Malkoc, S. and Bettman, J. (2009) Discounting time and time discounting: Subjective time perception and intertemporal preferences. Journal of Marketing Research, 46, 543-556. doi:10.1509/jmkr.46.4.543 